\title{
ArcheoSciences
}

Revue d'archéométrie

\section{Fluorescence UV des liants employés dans les dorures sur peintures murales médiévales}

UV Fluorescence of the Binding Media Used in Gilding of Mediaeval Mural Paintings

Aurélie Mounier, Laure Dayet, Floréal Daniel et Colette Belin

\section{(2) OpenEdition}

Journals

\section{Édition électronique}

URL : https://journals.openedition.org/archeosciences/2891

DOI : 10.4000/archeosciences.2891

ISBN : 978-2-7535-1849-0

ISSN : 2104-3728

Éditeur

Presses universitaires de Rennes

\section{Édition imprimée}

Date de publication : 30 avril 2011

Pagination : $19-28$

ISBN : 978-2-7535-1847-6

ISSN : $1960-1360$

\section{Référence électronique}

Aurélie Mounier, Laure Dayet, Floréal Daniel et Colette Belin, « Fluorescence UV des liants employés dans les dorures sur peintures murales médiévales », ArcheoSciences [En ligne], 35 | 2011, mis en ligne le 30 avril 2013, consulté le 28 février 2022. URL : http://journals.openedition.org/archeosciences/ 2891 ; DOI : https://doi.org/10.4000/archeosciences.2891 


\title{
Fluorescence UV des liants employés dans les dorures sur peintures murales médiévales
}

\author{
UV Fluorescence of the Binding Media Used in Gilding of Mediaeval Mural Paintings
}

\author{
Aurélie Mounier*, Laure DAYET*, Floréal DANIEL* et Colette Belin**
}

\begin{abstract}
Résumé : Les peintures de la chapelle de l’ancien logis abbatial de Moissac ont été examinées in situ sous éclairage UV. La présence de fluorescences jaunes sur les auréoles de certains personnages s'est révélée être l'indicateur de l'existence d'une ancienne dorure. Des fluorescences de ce type, caractéristiques d'un liant organique, ont déjà été observées dans d'autres sites où des traces de dorure sont également présentes. Une étude par spectrofluorimétrie montre que les spectres de fluorescence des deux types chimiques de liants les plus couramment employés dans la technique de la dorure sont différents. Pour se rapprocher de l'état d'altération des liants anciens, une étude de l'évolution de la fluorescence après vieillissement accéléré, a également été conduite.
\end{abstract}

\begin{abstract}
Paintings of the vault of the old abbey home of Moissac were examined using a UV source. The presence of yellow fluorescence on the halos figures is an indicator of the presence of historical gilding. Fluorescence of this type, characteristic of an organic binder, was already observed in other sites where traces of gilding are also present. A spectrofluorimetric study shows that the fluorescence spectra of the two chemical types of binders most usually used in the technique of gilding are different. To better understand the condition of aged binders, a study of the fluorescence evolution following accelerated ageing was also carried out.
\end{abstract}

Mots clé : fluorescences UV, liants lipidiques et protéiniques, dorure, peintures murales médiévales.

Keywords: UV fluorescences, lipidic, proteinaceous binders, gilding, Romanesque wall paintings.

\section{INTRODUCTION}

On a tort de considérer le décor médiéval et en particulier la peinture murale, une de ses expressions les plus caractéristiques, comme un art austère et entièrement soumis à la rigueur de la technique à la fresque. À l'époque romane les peintures étaient rehaussées de décors métalliques, d'incrustations de verre afin de donner plus de volume et de relief et d'ajouter à la couleur, reflets et brillance. Nous avons aujourd'hui, la plupart du temps, perdu ces décors et seule reste visible la couche sous-jacente à la feuille métallique, celle qui a permis l'adhésion du métal. C'est pourquoi l'étude des procédés de dorure est particulièrement intéressante d'un point de vue historique, en raison des critères non seulement esthétiques mais également symboliques et de prestige qui sont à l'origine de la réalisation des décors dorés.

Récemment, dans de nombreux sites de peintures datées $\mathrm{du} \mathrm{XI}{ }^{\mathrm{e}}$ au XIV ${ }^{\mathrm{e}}$ siècle, des traces d'anciennes dorures (à l'or,

* Institut de Recherche sur les Archéomatériaux (IRAMAT - CRPAA, UMR 5060), université Bordeaux - CNRS. (mounieraurelie33@yahoo.fr) (fdaniel@u-bordeaux3.fr)

** Institut des Sciences Moléculaires, UMR 5255, université de Bordeaux 1/CNRS, (c.belin@lpcm.u-bordeaux1.fr) 
l'argent ou l'étain) ont été mises au jour (Mounier et al., 2009a). Elles sont en général localisées dans des zones à forte charge symbolique : sur les auréoles de personnages importants (évangélistes, Christ, anges musiciens). C'est le cas des peintures de la chapelle de l'ancien logis abbatial de Moissac (Tarn-et-Garonne). On les trouve aussi sur des motifs décoratifs secondaires : les étoiles qui parsèment le fond des décors (absidiole sud de l'église Saint-Nicolas de Nogaro, Gers) ou bien les fleurettes et fleurs-de-lys sur le portail de la cathédrale Saint-Etienne de Cahors (Lot) (Czerniak et al., 2007).

Sur une même peinture, diverses techniques de dorure peuvent cohabiter. On en trouve la description dans les traités anciens (Pline l'Ancien ( $\mathrm{I}^{\mathrm{er}}$ siècle), Théophile ( $\mathrm{XII}^{\mathrm{e}}$ siècle), Cennino Cennini (XIv ${ }^{\mathrm{e}}$ siècle), Watin, (xvıII ${ }^{\mathrm{e}}$ siècle), etc.). On constate à la lecture de ces «manuels techniques » que de l'antiquité au XVIII ${ }^{\mathrm{e}}$ siècle, les modes d'application des décors métalliques ont subi peu de modifications. On dénombre deux principales techniques qui comportent des variantes et qui seront considérées ici :

- La dorure à la détrempe. Elle nécessite, pour faire adhérer la feuille métallique, l'emploi d'un liant organique de type colle protéinique (colle de peau, de nerf, d'os...) ou de gomme arabique.

- La dorure à la mixtion. Elle demande l'emploi d'un liant lipidique de type huile de lin, associé à de la céruse, par exemple, pour son pouvoir siccatif. Les analyses physicochimiques effectuées montrent que la technique à la mixtion est la plus courante (Katsibiri, 2004; Toniolo et al., 1998, Bonaduce, 2006).

Les décors métalliques ainsi apposés à sec, à l'aide d'un liant organique, présentent une plus grande fragilité et une moins bonne conservation. Il ne reste souvent de ces décors métalliques que quelques traces car leur mode d'application les rend sensibles aux nombreux facteurs de dégradation, notamment environnementaux, auxquels ils ont été soumis au cours des siècles. Le plus souvent, il n'en subsiste que les résidus de liants organiques qui ont servi à faire adhérer la feuille métallique. C'est par leur détection qu'il nous est possible aujourd'hui de situer l'emplacement d'une dorure ancienne. C'est, par exemple, grâce à la fluorescence jaune provoquée par un éclairage sous lampe UV (fig. 1) que des traces de dorure ont été découvertes récemment dans certaines peintures murales du sud-ouest de la France (Mounier et al., 2009a).

En ce qui concerne les métaux, l'or, l'étain, l'argent ont été utilisés (Nadolny, 2006). L'étain semble être le plus économique et peut être employé sous l'or ou recouvert d'un vernis jaune pour imiter l'or. Le choix de l'étain à la place de l'argent s'explique par ses meilleures qualités de conservation (Mounier et al., 2009b).

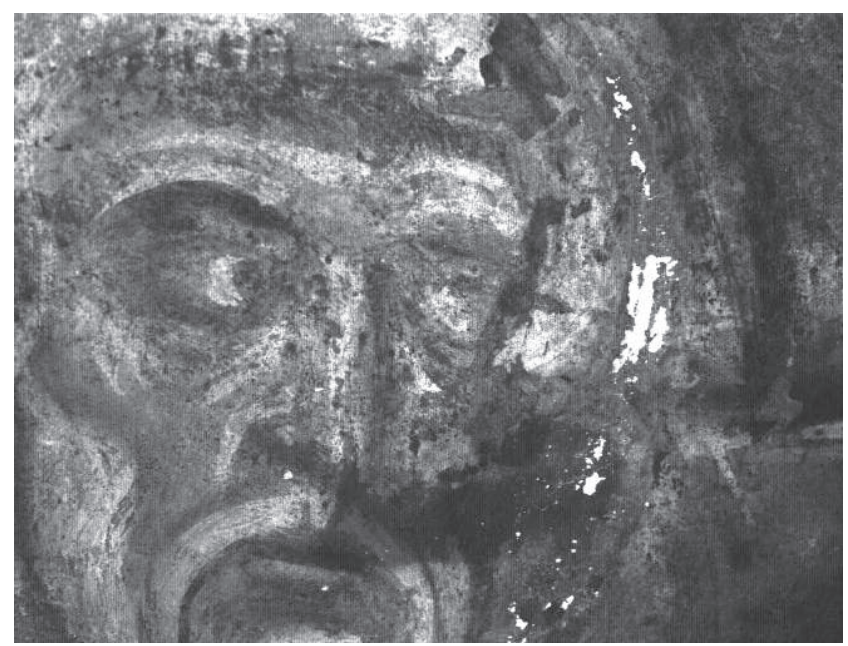

Figure 1: (Voir planche couleur) Fluorescence jaune obtenue sous UV grâce à une caméra multispectrale sur l'auréole d'un des personnages des peintures murales de la voûte de la chapelle de l'ancien logis abbatial de Moissac (xir ${ }^{\text {e }}$ siècle Tarn-etGaronne). L'aspect bleuté de l'image correspond à la réflexion de la lumière ultraviolette par le support et non à une fluorescence.

Figure 1: (See colour plate) Yellow fluorescence obtained under UV with a multispectral camera on the halo of one of the characters on the mural paintings of the chapel vault of the ancient abbey home in Moissac (12th c., Tarn-et-Garonne). The bluish aspect of the image corresponds to the ultraviolet light reflexion by the support and not to a fluorescence.

D'un point de vue chimique, les liants utilisés dans la peinture sont donc de quatre types : protéinique, lipidique, polysaccharidique, ou un mélange lipides/protéines. Les plus utilisés dans les dorures sont les deux premiers : liants protéiniques, de type colle d'os ou colle de nerf constitués plus particulièrement de molécules de collagène et des dérivés de cette protéine. D'autres types de colles protéiniques existent, notamment la caséine ou le blanc d'œuf; liants lipidiques, les huiles siccatives sont des triglycérides, c'està-dire des esters du glycérol (un triple alcool) et d'acides gras (acides à longue chaîne carbonée) (Masschelein-Kleiner, 1992; Perego, 2005).

Les analyses faites sur des échantillons d'huile de lin montrent que l'huile fraîche produit un signal de fluorescence faible, avec une longueur d'onde maximale proche de 470480 nm (De la Rie, 1982b; Chryssoulakis, 1989). En séchant, l'intensité de fluorescence a tendance à augmenter lorsque l'huile est placée dans l'obscurité ou chauffée à $100^{\circ} \mathrm{C}$, et la longueur d'onde maximale devient supérieure à $500 \mathrm{~nm}$ (De la Rie, 1982b; Mallégol et al., 2001). La longueur d'onde d'émission des protéines semble être toujours inférieure à 
470 nm (Larson et al., 1991; Nevin et al., 2006; Nevin et al., 2007). Le jaune d'œuf vieilli (préparation en 1937) présente un maximum de fluorescence supérieur, centré vers $580 \mathrm{~nm}$ (Larson et al., 1991). Les changements observés sur la longueur d'onde maximale après des temps de séchage différents ( 3 mois, 4 mois ou 1 an) sont peu marqués (Nevin et Anglos, 2006, Nevin et al., 2006; Nevin et al., 2007). Les travaux réalisés dans ce domaine sont récents (Thoury et al., 2007; Nevin et al., 2008). Ils traitent des conséquences des altérations chimiques des liants sur l'émission de fluorescence. Les résultats permettent, par exemple, d'identifier des vernis utilisés en restauration en comparant le spectre UV de fluorescence à celui de vernis connus et vieillis artificiellement (Thoury et al., 2007). Une autre étude a été mise en œuvre afin de différencier les liants protéiniques employés en peinture (œuf, blanc d'œuf et jaune d'œuf, lait et caséine) ainsi que les colles à base de collagène (peau de lapin, os, parchemin et colle de poisson [Nevin et al., 2008]).

À la lumière des résultats déjà publiés et par comparaison avec notre expérience sur l'examen in situ de décors métalliques dans les peintures murales médiévales, il paraissait intéressant de mener une étude, dont les objectifs sont essentiellement méthodologiques, à propos de la fluorescence des différentes familles chimiques de liants employés dans les techniques de dorure et de l'influence du vieillissement artificiel sur leurs propriétés de fluorescence afin de répondre aux questions suivantes :
Peut-on par observation UV in situ, retrouver des zones anciennement dorées grâce au liant résiduel? La couleur de la fluorescence peut-elle nous renseigner sans ambiguïté sur la technique de dorure employée?

Lidentification et l'analyse d'une dorure sur une peinture apportent des informations importantes. À l'historien d'art, elle permet de hiérarchiser les différents éléments de l'iconographie. Au restaurateur, la connaissance de la nature des matériaux utilisés (feuille d'or ou d'argent, collée avec de l'huile de lin, de la cire ou une colle animale) oriente ses choix dans les traitements et les produits à appliquer pour la préservation de ces décors.

\section{EXPÉRIMENTAL}

\section{Échantillons}

Des échantillons de liants purs ont été déposés sous forme de films (sur des lames de verre). Le tableau 1 résume l'ensemble des échantillons étudiés (un choix de neuf types de liants parmi les plus utilisés à l'époque médiévale pour la réalisation de dorures).

Les liants purs ont été achetés dans le commerce. La colle d'os (colle d'os en perles), la colle de nerf (colle de nerfs en grains), la caséine, la gomme arabique brute et l'huile de noix ont été fournies par la société Okhra (Roussillon).

\begin{tabular}{|c|c|c|c|c|}
\hline & Liants & $\lambda \max ($ bibliographie) en $\mathrm{nm}$ & $\begin{array}{l}\text { Nos liants de référence } \\
\text { et fournisseur }\end{array}$ & $\lambda \max (\mathrm{nm})$ mesurée \\
\hline \multirow{3}{*}{$\frac{\mathscr{8}}{\mathscr{n}}:$} & Huile de lin & $\begin{array}{l}540 \\
\text { (Chryssoulakis et al., 1989; Larson et al., 1991) }\end{array}$ & $\begin{array}{l}\text { Huile de lin clarifiée Lefranc et } \\
\text { Bourgeois }\end{array}$ & 545 \\
\hline & \multirow{2}{*}{\multicolumn{2}{|c|}{ Jaune d'œuf }} & $\begin{array}{l}\text { Huile de noix } \\
\text { Okhra }\end{array}$ & 526 \\
\hline & & & 555 & \\
\hline \multirow{4}{*}{ 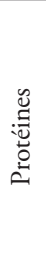 } & Blanc d'œuf & $\begin{array}{l}\text { 420-440 } \\
\text { (Nevin et al., 2006; 2007; 2008) }\end{array}$ & Blanc d'œuf & 433 \\
\hline & \multirow[t]{2}{*}{ Colles protéiniques } & \multirow{2}{*}{$\begin{array}{l}\text { 400-475 } \\
\text { (Chryssoulakis et al., } 1989 \text {; Nevin et al., } 2006 ; 2007 ; 2008)\end{array}$} & $\begin{array}{l}\text { Colle d'os } \\
\text { Okhra }\end{array}$ & 435 \\
\hline & & & Colle de nerf en grains Okhra & 436 \\
\hline & Caséine & $\begin{array}{l}435 \\
\text { (Larson et al., } 1991 \text {; Nevin et al., 2006) }\end{array}$ & $\begin{array}{l}\text { Caséine } \\
\text { Okhra }\end{array}$ & 456 \\
\hline 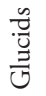 & Gomme arabique & $\begin{array}{l}451 \\
\text { (Larson et al., 1991) }\end{array}$ & $\begin{array}{l}\text { Gomme arabique } \\
\text { Okhra }\end{array}$ & 439 \\
\hline
\end{tabular}

Tableau 1 : Longueur d'onde maximale donnée par les analyses de fluorimétrie sur les liants purs. On constate que la longueur d'onde d'émission maximale pour les lipides est supérieure à $500 \mathrm{~nm}$ et pour les protéines, inférieure à $450 \mathrm{~nm}$.

Table 1: Maximum wavelength given by fluorimetric analyses on pure binders. It is noted that the maximum emission wavelength for the lipids is higher than $500 \mathrm{~nm}$ and for the proteins lower than $450 \mathrm{~nm}$. 
L'huile de lin (huile de lin clarifiée) provient de la société Lefranc et Bourgeois (Le Mans).

Une première série de films a été laissée à sécher. Le " séchage " des huiles correspond à une réticulation et demande plus de temps que les liants protéiniques, c'est pourquoi elles ont été laissées à vieillir naturellement pendant deux ans. Quatre séries de liants purs sur lame de verre ont été préparées. Des vieillissements accélérés de ces liants ont été réalisés selon deux protocoles différents (thermohygrométrique [V1] ou sous UV [V2]) qui seront décrits plus loin. Les échantillons non vieillis sont codifiés V0.

\section{Méthodes}

\section{Fluorescence UV}

L'étude expérimentale de la fluorescence des liants organiques en fonction de leur nature chimique et de leur état de dégradation est réalisée à l'aide d'un spectrofluorimètre de laboratoire SPEX Fluorolog, modèle 212 qui se compose d'une source arc xénon de $450 \mathrm{~W}$, donnant un rayonnement polychrome continu, d'un double monochromateur constitué de réseaux plans pour l'excitation, d'un compartiment porte-échantillon, d'un second double monochromateur à réseaux pour l'émission, d'un photomultiplicateur. Les paramètres d'acquisition choisis sont les suivants : angle de $22,5^{\circ}$ entre le faisceau incident et le détecteur; excitatrice de $366 \mathrm{~nm}$, mode à flux excitateur constant. La largeur de fente est de 2,2 $\mathrm{mm}$ ce qui correspond à une bande passante de $4 \mathrm{~nm}$, pour un pas de $0,5 \mathrm{~nm}$ et un temps d'intégration de 0,5 seconde. Aux spectres obtenus ont été appliqués une fonction corrective, ce qui permet de s'affranchir des contraintes liées à l'instrumentation. La calibration est réalisée avec une substance fluorescente (Rhodamine).

Le choix des conditions opératoires a été déterminé par la nécessité de comparer les informations obtenues par d'autres moyens d'examen de la fluorescence des liants :

- l'examen in situ réalisé à l'aide d'une caméra multispectrale ARTIST (Art Innovation) $(\lambda=365 \mathrm{~nm})$. La source UV est une lampe à mercure avec une irradiation UV comprise entre 3000 et $90000 \mu \mathrm{W} / \mathrm{cm}^{2}$ qui dépend de la distance de travail.

- une source UV montée sur un microscope optique permettant l'examen des prélèvements et, en particulier, de leur coupe stratigraphique. La source Exfo X-Cite series 120PC est une lampe au mercure. Le filtre d'excitation permet de sélectionner une bande d'UV de 360 à $370 \mathrm{~nm}$ et un autre filtre avec une longueur d'onde inférieure à $400 \mathrm{~nm}$ permet d'éliminer la lumière émise par l'échantillon.
Pour la mise en œuvre, divers paramètres sont à prendre en compte et doivent être les plus proches possibles pour comparer et expliquer les résultats :

a) $\lambda$ d'excitation : La source UV $(\lambda=365 \mathrm{~nm})$ utilisée in situ en association avec la caméra multispectrale est comparable à celle du spectrofluorimètre $(362-366 \mathrm{~nm})$ et à celle couplée à un microscope optique (360-370 nm).

b) Zone analysée : elle varie énormément d'un appareil à l'autre : $0,8 \times 2 \mathrm{~mm}$ pour le spectrofluorimètre; $-50 \mathrm{~cm}^{2}$ pour la caméra multispectrale et de l'ordre du micromètre au microscope. La zone d'analyse doit être considérée car selon la méthode employée, on ne travaille pas à la même échelle.

Pour l'exploitation des données seront prises en considération la longueur d'onde à l'intensité maximale $\left(\lambda_{\max }\right)$ et la couleur. L' $\lambda_{\max }$, est un caractère quantifiable très souvent utilisé pour comparer des spectres de fluorescence entre eux. Les longueurs d'onde correspondant au maximum d'intensité ont été déterminées pour chaque liant, ainsi que le domaine de couleur correspondant (tableau 1 et 2).

\section{Vieillissements artificiels}

1- Le vieillissement thermo-hygrométrique des échantillons (sur lames de verre) a été réalisé dans une enceinte climatique (Vötsch VC 4018). Le protocole utilisé comprend des cycles de huit heures qui comportent quatre phases de 90 minutes, avec des montées et descentes de trente minutes entre chaque cycle selon un protocole utilisé pour le vieillissement accéléré de pigments (Aze, 2005). Les différentes phases et les conditions de température et humidité relative suivies dans ce protocole sont les suivantes :

\begin{tabular}{|l|l|l|l|}
\hline & L$^{*}$ & $\mathbf{a}^{*}$ & $\mathbf{b}^{*}$ \\
\hline Lipides & & & \\
\hline Huile de lin & 93,2 & 29,7 & 28,1 \\
\hline Huile de noix & 88,5 & 37,2 & 7,5 \\
\hline Jaune d'œuf & 85,9 & 44,0 & 60,3 \\
\hline Mélange & & & \\
\hline Euf entier & 51,4 & $-0,2$ & $-55,0$ \\
\hline Protéines & & & \\
\hline Blanc d'œuf & 50,7 & $-30,7$ & $-65,8$ \\
\hline Colle d'os & 62,8 & $-37,9$ & $-56,8$ \\
\hline Colle de nerf & 57,8 & $-2,5$ & $-62,7$ \\
\hline Caséine & 48,6 & $-1,3$ & $-45,5$ \\
\hline
\end{tabular}

Tableau 2 : Coordonnées chromatiques déterminées à partir du spectre de fluorescence UV des différents liants purs.

Table 2: Given chromatics coordinates from the UV fluorescence spectrum of the various pure binders. 
- une phase de forte humidité : température $(\mathrm{T})$ de $18^{\circ} \mathrm{C}$ et une humidité relative (HR) de $85 \%$

- une phase de basse température : $\mathrm{T}=-10^{\circ} \mathrm{C}, \mathrm{HR}=0 \%$

- une phase de chaleur sèche : $\mathrm{T}=40^{\circ} \mathrm{C}, \mathrm{HR}=25 \%$

- une phase de chaleur humide $: \mathrm{T}=30^{\circ} \mathrm{C}, \mathrm{HR}=60 \%$

Ce cycle de huit heures a été répété 90 fois pour une durée totale de quatre semaines.

2- Le vieillissement artificiel par exposition aux rayonnements UV a été réalisé dans une enceinte qui permet d'irradier les échantillons par des UV B de longueur d'onde $313 \mathrm{~nm}$ et les maintient à une température constante de $45^{\circ} \mathrm{C}$ (QUV Accelerated Weathering Tester). L'irradiance de la source est de $0,71 \mathrm{~W} / \mathrm{m}^{2}$ lorsque les échantillons sont situés à la distance de $4,5 \mathrm{~cm}$. L'exposition aux UV a duré 400 heures au total, soit environ 17 jours.

\section{Résultats}

\section{$\lambda_{\text {max }}$ de l'émission de fluorescence des liants avant vieillissement}

Certaines limitations de la méthode doivent être prises en compte. Certains pigments sont des inhibiteurs de fluorescence (les ocres, notamment), d'autres au contraire apportent une fluorescence supplémentaire. C'est pourquoi il est difficile de travailler sur des mélanges. Il faut tenir compte également de la fluorescence du support. La comparaison de la fluorescence des liants sur des lames de verre et sur pierre calcaire montre que l'on n'observe pas les mêmes résultats. Le calcaire a sa propre émission de fluorescence (rose violet) qui varie selon sa teneur en eau. On peut penser que la fluorescence du support a une influence sur la fluorescence émise finale observée.

Selon la bibliographie citée plus haut, les huiles sèches ont une $\lambda_{\text {max }}>500 \mathrm{~nm}$, les protéines et les glucides ont une $\lambda_{\text {max }}$ $<450 \mathrm{~nm}$, ce qui est confirmé par nos résultats (tableau 1) obtenus sur les colles protéiniques, la gomme arabique et les huiles sèches. Nous avons, en plus, mené notre étude sur de l'œuf. La partie la plus riche en lipides (jaune d'œuf) a une $\lambda_{\max }>500 \mathrm{~nm}$, la plus riche en protéines (blanc d'œuf) est inférieure à $450 \mathrm{~nm}$.

Deux groupes parmi les liants non vieillis se différencient (tableau 1) :

- Les liants protéiniques (caséine, colles et blanc d'œuf), la gomme arabique et l'huile de noix ont une émission de fluorescence dans le bleu-pourpré, avec une longueur d'onde comprise entre 430 et $456 \mathrm{~nm}$. L'œuf entier peut également être placé dans ce groupe, avec une longueur d'onde maximale d'émission à $423 \mathrm{~nm}$, dans le domaine du violet.
- L'huile de lin et le jaune d'œuf ont une $\lambda_{\text {max }}$ supérieure à $500 \mathrm{~nm}$, c'est à dire dans le domaine du vert et vert-jaune.

L'émission de fluorescence du jaune d'œuf est connue et due à la présence des acides aminés aromatiques des protéines, des phospholipides et des produits dérivés. Les protéines contiennent trois acides aminés qui contribuent à la fluorescence UV : la tyrosine, la phénylalanine et le tryptophane. L'observation de l'émission de fluorescence du jaune d'œuf excité à $436 \mathrm{~nm}$ montre, par exemple, un maximum de fluorescence entre 520 et $570 \mathrm{~nm}$ (Gaspard et al., 2008). Dans notre étude, le jaune d'œuf excité à $366 \mathrm{~nm}$ montre un maximum d'émission de fluorescence centré à $555 \mathrm{~nm}$, ce qui va dans le même sens que la bibliographie (figure 2).

Ces résultats sont cohérents avec ce que nous trouvons dans la littérature, en particulier pour les protéines, excepté toutefois pour le jaune d'œuf (Castillejo et al., 2002). Nous avons constaté cependant que pour ce dernier, l'épaisseur du film à une influence sur l'intensité relative des différentes bandes du spectre. Une étude plus poussée serait nécessaire pour mieux comprendre les différences entre nos résultats et ceux présentés dans les études antérieures.

La fluorimétrie est donc un moyen fiable de discriminer les grandes familles de liants. Les protéines et les lipides n'ont pas la même allure de spectre d'émission de fluorescence et leurs longueurs d'onde maximales sont différentes.

\section{Correspondance du spectre d'émission de fluorescence en coordonnées chromatiques}

Dans la mesure où nous cherchons à mettre en évidence des variations de couleur, des diagrammes de chromaticité $a^{*} b^{*}$ ont été réalisés pour l'ensemble des liants. L'avantage

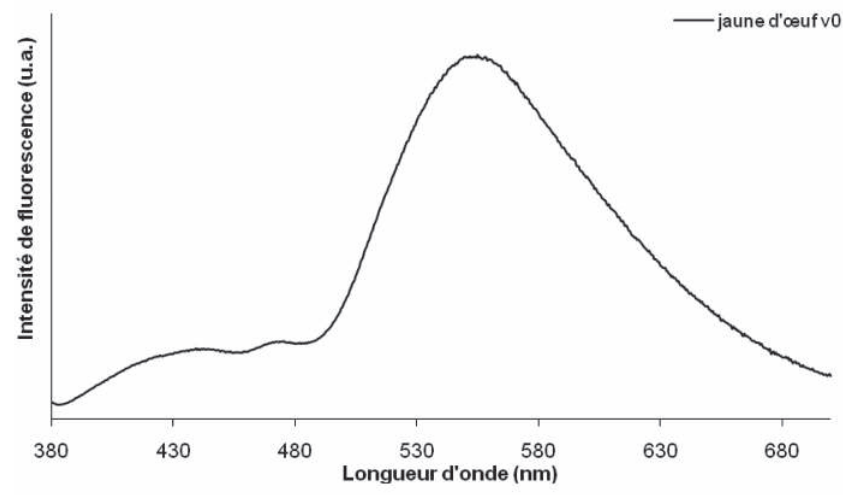

Figure 2 : Spectre d'émission de fluorescence du jaune d'œuf avant vieillissement. Le maximum de fluorescence est centré vers 555 $\mathrm{nm}$.

Figure 2: Emission fluorescence spectrum of the egg yolk before ageing. The fluorescence maximum is about $555 \mathrm{~nm}$. 
de ces diagrammes est de prendre en compte l'ensemble du spectre ${ }^{1}$. Ils semblent plus appropriés que la longueur d'onde pour définir ce que l'on voit visuellement et permet de donner la gamme chromatique de la fluorescence. À l'intérieur de ces diagrammes, les échantillons se distinguent par une valeur de $b^{*}$ positive (jaune) ou négative (bleu), $a^{*}$ étant toujours négative (vert).

Si l'on transpose les spectres d'émission de fluorescence en coordonnées chromatiques $\mathrm{L}^{*} \mathrm{a}^{*} \mathrm{~b}^{*}$, deux grandes familles se distinguent : les huiles (dans le quart jaune vert) et les protéines (dans le quart vert bleu) (figure 3).

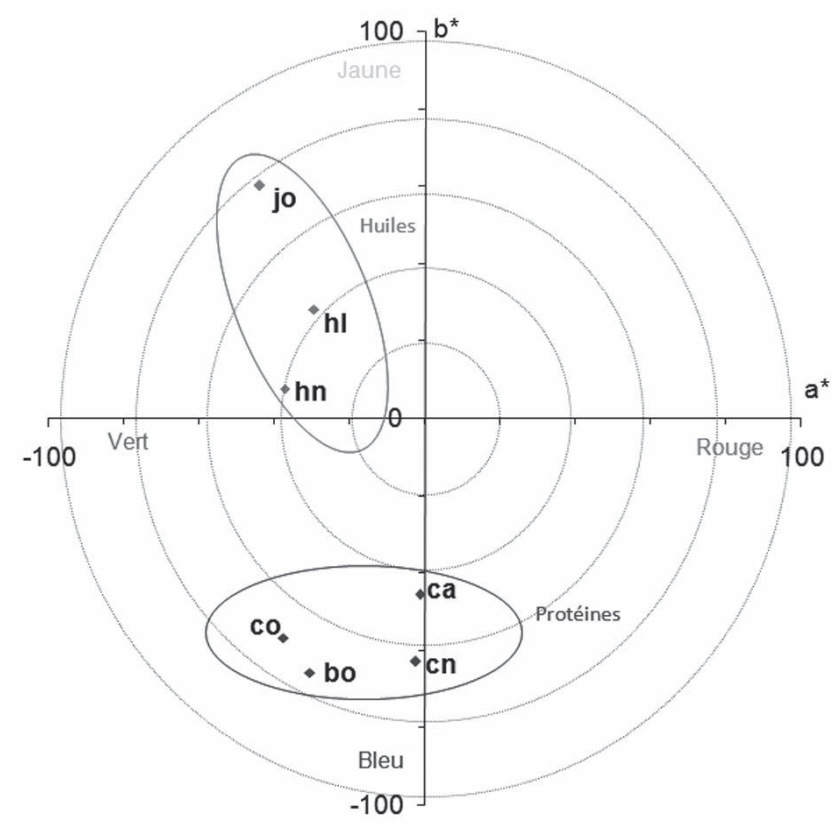

Figure 3 : (Voir planche couleur) Diagramme de chromaticité des longueurs d'ondes maximales des différents liants étudiés. Les huiles (jo : jaune d'œuf; hl : huile de lin; hn : huile de noix) fluorescent avec une $\lambda$ max dans le jaune-vert. Les protéines (ca : caséine; cn : colle de nerf; co : colle d'os; bo : blanc d'œuf) avec une $\lambda$ max dans le bleu.

Figure 3: (See colour plate) Chromaticity diagram of maxima wavelengths of the various studied binders. The oils (jo: egg yolk; hl: linseed oil; hn: walnut oil) give a fluorescence with a $\lambda$ max in yellow-green. The proteins (ca: casein; cn: nerve glue; co: bone glue; bo: egg white) with a $\lambda$ max in blue.

1. Les données du spectre de fluorescence ont été reportées dans le logiciel de traitement de spectres d'un spectrocolorimètre Minolta CS-S1W, qui à partir de l'ensemble du spectre, fournit les coordonnées chromatiques correspondantes ( $L^{*}, a^{*}, b^{*}$ ou Xxy).

\section{Influence du vieillissement sur l'émission et la couleur de la fluorescence des liants}

Dans l'ensemble, les vieillissements n'ont que très peu d'effets pour les colles et la gomme arabique, la $\lambda_{\text {max }}$ ne variant pas de plus de $20 \mathrm{~nm}$ (tableau 3). Pour le blanc d'œuf, l'œuf et la caséine, on note un déplacement de la longueur d'onde maximale vers les grandes longueurs d'onde et un élargissement de la bande d'émission pour certains protocoles de vieillissement. Pour tous ces liants, la longueur d'onde maximale est toujours inférieure à $500 \mathrm{~nm}$, et comprise dans les domaines du bleu pourpré au bleu-vert.

Le vieillissement à la lumière produit le plus d'effet sur le déplacement de la longueur d'onde maximale ainsi que sur la couleur (tableau 4). Le vieillissement thermo hygrométrique semble avoir peu d'effets. $L^{*}$ n'a pas été pris en compte car il est fonction de l'épaisseur du film de colle. Celui-ci n'était pas constant et uniforme entre les éprouvettes. Les coordonnées chromatiques $\mathrm{a}^{*}$ et $\mathrm{b}^{*}$ sont en relation avec la couleur de l'émission de fluorescence observée (donc avec les données de l'imagerie). Ce sont ces paramètres que nous avons choisi d'exploiter.

La tendance générale montre un déplacement des coordonnées chromatiques après vieillissement. La colle d'os semble moins sensible à ces types de vieillissement. La caséine est la protéine qui subit le plus d'évolution. La fluorescence émise dans l'espace chromatique du bleu des protéines tend vers le vert après vieillissement accéléré (figure 4).

\begin{tabular}{|l|c|c|c|}
\hline & Non vieillis (V0) & $\begin{array}{c}\text { Vieillis T, HR } \\
\text { (V1) }\end{array}$ & $\begin{array}{c}\text { Vieillis lumière } \\
\text { (V2) }\end{array}$ \\
\hline Lipides & & & - \\
\hline Huile de lin & 545 & - & - \\
\hline Huile de noix & 526 & - & - \\
\hline Jaune d'ouf & 555 & - & 470 \\
\hline Protéines & & & 459 \\
\hline Blanc d'ouf & 433 & 434 & 451 \\
\hline Colle d'os & 435 & 450 & 469 \\
\hline Colle de nerf & 436 & 434 & \\
\hline Caséine & 456 & 496 & - \\
\hline Glucides & & 450 & \\
\hline $\begin{array}{l}\text { Gomme ara- } \\
\text { bique }\end{array}$ & 439 & & \\
\hline
\end{tabular}

Tableau 3 : Longueurs d'onde maximales de fluorescence des liants frais (V0) sur lame de verre. Longueurs d'onde maximales d'émission des liants sur lame de verre vieillis (V1 : Vieillissement thermo-hygrométrique; V2 : Vieillissement sous UV).

Table 3: Maximum wavelength fluorescence of the pure binders (VO) on glass slide. Maximum emission wavelength of binders on glass slide aged (V1: Hygro-thermal ageing; V2: UV lignt ageing). 


\begin{tabular}{|c|c|c|c|c|c|c|c|c|c|}
\hline & \multicolumn{4}{|c|}{ V0 } & \multicolumn{3}{c|}{ V1 (Vacc Temp) } & \multicolumn{3}{c|}{ V2 (Vacc Lumière) } \\
\hline & $\mathrm{L}^{*}$ & $\mathrm{a}^{*}$ & $\mathrm{~b}^{*}$ & $\mathrm{~L}^{*}$ & $\mathrm{a}^{*}$ & $\mathrm{~b}^{*}$ & $\mathrm{~L}^{*}$ & $\mathrm{a}^{*}$ & $\mathrm{~b}^{*}$ \\
\hline Colle d'os & 62,8 & $-37,9$ & $-56,8$ & 61,7 & $-36,5$ & $-58,3$ & 67,0 & $-38,4$ & $-49,5$ \\
\hline $\begin{array}{c}\text { Colle de } \\
\text { nerfs }\end{array}$ & 57,8 & $-2,5$ & $-62,7$ & 61,6 & $-6,6$ & $-57,6$ & 68,5 & $-18,4$ & $-48,6$ \\
\hline Caséine & 48,6 & $-1,3$ & $-45,5$ & 84,2 & 35,9 & 20,0 & 77,8 & $-35,3$ & $-32,4$ \\
\hline
\end{tabular}

Tableau 4 : Coordonnées chromatiques déterminés à partir du spectre de fluorescence UV de trois protéines (colle d'os, colle de nerf et caséine) témoins, vieillies par température et humidité relative et à la lumière.

Table 4: Chromatics coordinates determined from the UV fluorescence spectrum of three proteins (bone glue, nerve glue and casein) references, aged by temperature and relative humidity and by UV light.

Il semble que les protocoles de vieillissement, et donc les facteurs environnementaux que l'on fait varier, favorisent plus ou moins la fluorescence de l'un ou de l'autre des composés fluorescents. L'influence de l'épaisseur des films ne doit pas non plus être négligée ${ }^{2}$.

On n'observe pas d'effet significatif du vieillissement thermique sur la longueur d'onde maximale de l'émission de fluorescence des protéines, sauf peut-être un léger décalage lorsqu'elles ont été soumises au vieillissement à la lumière (figure 5).

En ce qui concerne les huiles, selon la bibliographie, on s'attend à des modifications qui ont, du reste, été constatées par FTIR (avec la disparition des bandes C-H, ce qui correspond à une réticulation ${ }^{3}$ ). Les vieillissements artificiels devraient provoquer un déplacement de la longueur d'onde maximale vers des valeurs plus élevées (effet bathochrome).

\section{Observation de la dégradation des liants par spectrométrie infrarouge}

Pour l'ensemble des liants, frais et vieillis, des spectres d'absorption infrarouge ont été enregistrés, dans le but d'étudier les dégradations des liants.

Sur les spectres des colles notamment, on observe des changements sur les bandes vers 3290-3270 $\mathrm{cm}^{-1}(v \mathrm{~N}-\mathrm{H})$, et celles vers $1540-1530 \mathrm{~cm}^{-1}(\delta \mathrm{N}-\mathrm{H}$ et $v \mathrm{C}-\mathrm{N})$. Ces bandes sont caractéristiques du collagène. Le décalage de ces bandes semble indiquer une dénaturation plus poussée du collagène et de ses dérivés, en particulier une désorganisation de leur structure (Rabotyagova et al., 2008; Wisniewski et al., 2007).

En ce qui concerne l'huile de lin, on note des changements sur les bandes vers 2950-2850 $\mathrm{cm}^{-1}(v \mathrm{C}-\mathrm{H})$ et vers 1750-1720 $\mathrm{cm}^{-1}(v \mathrm{C}=\mathrm{O})$. La diminution d'intensité des

2. Donnée non prise en compte dans cette étude

3. Phénomène d'oxydation des acides gras insaturés d'une huile siccative conduisant à la formation d'un réseau tridimensionnel.

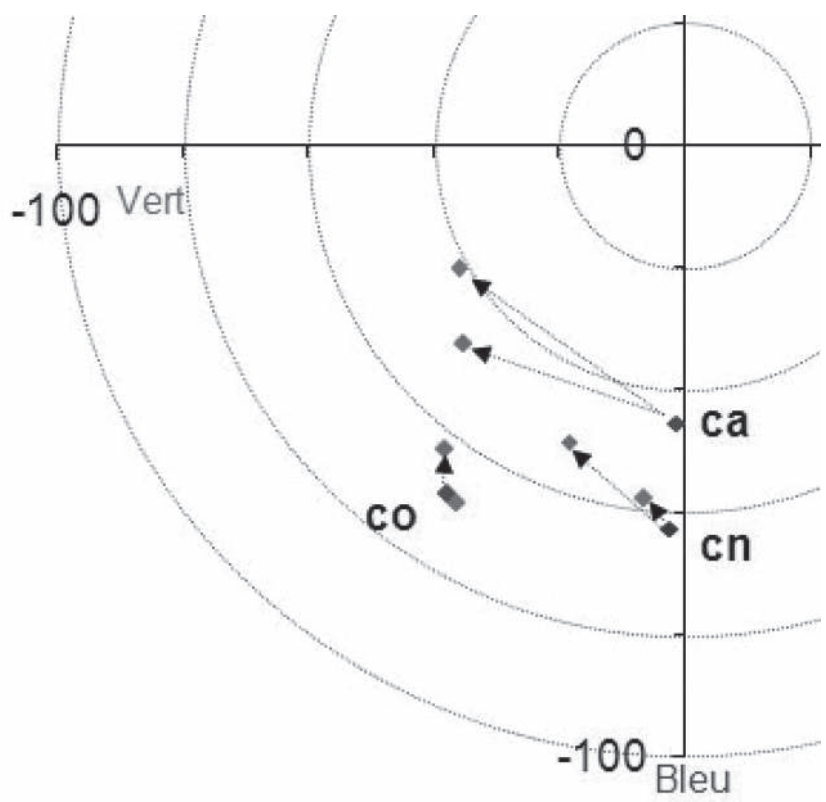

Figure 4 : (Voir planche couleur) Diagramme chromatique montrant l'évolution de la longueur d'onde maximale après vieillissement. On note un léger déplacement des longueurs d'ondes maximales des protéines (ca : caséine; cn : colle de nerf; co : colle d'os) du bleu vers le vert (effet bathochrome). Ceci est encore plus marquant au vieillissement à la lumière. Figure 4: (See colour plate) Chromatic diagram showing the maximum wavelength evolution after ageing. We notice a weak displacement of the proteins maxima wavelength (ca: casein; cn: nerve glue; co: bone glue) blue towards the green (bathochrome effect). This is even more outstanding with the light ageing.

bandes de vibration $v \mathrm{C}-\mathrm{H}$ corrélée au décalage de la bande de vibration $v \mathrm{C}=\mathrm{O}$ de $1740 \mathrm{~cm}^{-1}$ environ à $1720 \mathrm{~cm}^{-1}$, (Figure 6) nous indique que des phénomènes d'oxydation importants se sont produits, avec sans doute l'apparition de nouveaux groupements carbonyles $(\mathrm{C}=\mathrm{O})$ et une disparition progressive des liaisons esters (Mallégol et al., 2000). 


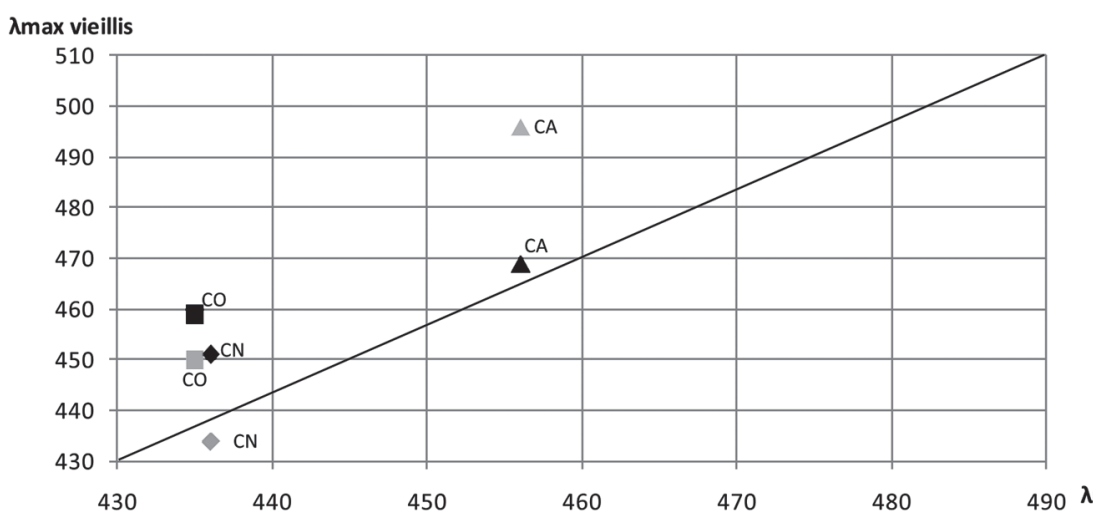

Figure 5: Graphique représentant l'influence du vieillissement accéléré (thermique en gris clair et à la lumière en noir) sur la longueur d'onde maximale de trois liants protéiniques (colle de nerf : $\mathrm{CN}$; colle d'os : $\mathrm{CO}$ et caséine : $\mathrm{CA}$ ). Au dessus de la diagonale, la longueur d'onde maximale augmente après le vieillissement.

Figure 5: Graphic representing the accelerated ageing influence (thermal in light grey and with the light in black) on the maximum wavelength of three proteinics binders (nerve glue: $C N$; bone glue: $C O$ and casein: $C A$ ). Above the diagonal, the maximum wavelength increases after the ageing.

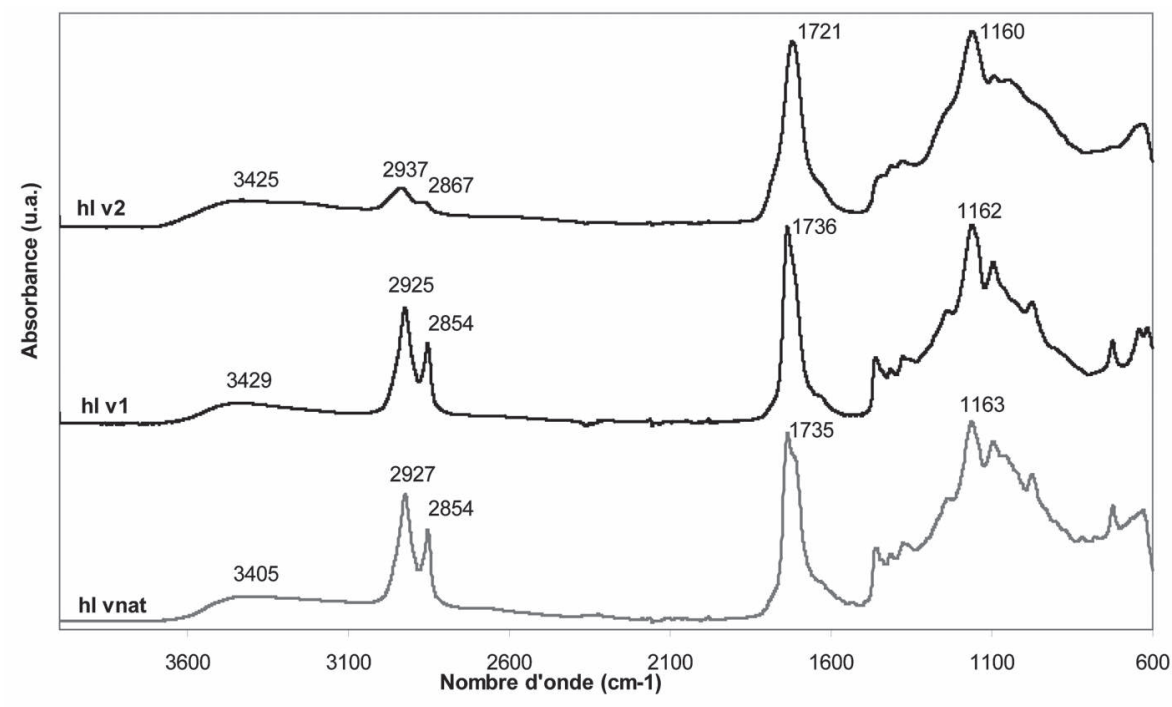

Figure 6: Spectres infrarouge de l'huile de lin, échantillon de référence (v0) et échantillons vieillis (vnat, v1, v2). On note des changements sur les bandes $(v \mathrm{C}-\mathrm{H})$ vers $2950-2850 \mathrm{~cm}^{-1}$ et vers $1750-1720 \mathrm{~cm}^{-1}(v \mathrm{C}=\mathrm{O})$. La diminution d'intensité des $v \mathrm{C}-\mathrm{H}$ corrélée au décalage de la bande de vibration $v \mathrm{C}=\mathrm{O}$ de $1740 \mathrm{~cm}^{-1}$ environ à $1720 \mathrm{~cm}^{-1}$, indiquent des phénomènes d'oxydation.

Figure 6: Infrared spectra of the linseed oil, reference sample $(v 0)$ and aged samples (vnat, v1, v2). We notice modifications on the bands (vC-H) towards 2950-2850 $\mathrm{cm}^{-1}$ and towards $1750-1720 \mathrm{~cm}^{-1}(\mathrm{v} C=O)$. The intensity decrease of $v C-H$ correlated with the vibration band gap $v C=O$ of about $1740 \mathrm{~cm}^{-1}$ to $1720 \mathrm{~cm}^{-1}$, indicate oxidation phenomena.

\section{Conclusion}

L'observation sous UV permet, dans certains cas, de situer les zones où un décor métallique, aujourd'hui disparu ou altéré, a pu exister. La fluorescence est spécifique à la famille chimique du liant qui est, elle-même, caractéristique de la technique. Ceci a été vérifié par spectrofluorimétrie.

L'examen in situ à l'aide d'une caméra multispectrale, les observations sous microscope optique et sous UV de coupes stratigraphiques et l'analyse par spectrofluorimétrie apportent donc des informations complémentaires entre elles et vont dans le même sens. Après vieillissement, les liants voient leur longueur d'onde maximale augmenter. Pour l'huile, le vieillissement à la lumière accélère le processus d'oxydation et a le plus d'effet.
Des vieillissements plus longs peuvent être envisagés afin d'approfondir l'étude de la fluorescence et de la dégradation des liants, notamment pour les huiles. L'étude de mélanges (huile + blanc de plomb) est en cours afin de connaître les effets des siccatifs sur la fluorescence. L'association de liants à différents pigments (ocre rouge, cinabre, azurite) est également une piste à exploiter car on suppose que le pigment a un rôle non négligeable dans la florescence observée.

Létude de la fluorescence UV de liants frais et de liants vieillis nous a permis de déterminer des groupes de liants, en prenant en compte la longueur d'onde maximale d'une part, et les coordonnées chromatiques $\mathrm{a}^{*}$ et $\mathrm{b}^{*}$ d'autre part. L'une comme l'autre permettent d'observer les variations de fluorescence de façon simple et efficace. Les diagrammes de chromaticité, toutefois, rendent compte de l'ensemble des spectres, ils sont donc plus pertinents que la longueur 
d'onde maximale pour comparer les domaines chromatiques de la fluorescence (bleu-vert pour les protéines, œuf et gomme arabique $\lambda<500 \mathrm{~nm}$; vert-jaune pour les huiles $\lambda>520 \mathrm{~nm})$.

\section{Remerciements}

Merci à Thierry Buffeteau de l'Institut des Sciences Moléculaires de l'université de Bordeaux 1, qui nous a donné accès au spectromètre infrarouge à transformée de Fourier.

\section{Bibliographie}

AzE, S., 2005. Altérations chromatiques des pigments au plomb dans les auvres du patrimoine - Étude expérimentale des altérations observées sur les peintures murales, Thèse de doctorat, Université de droit, d'économie et des sciences d'Aix-Marseille, p. 82-83.

Bonaduce I., Colombani M.-P. and Diring S., 2006 Identification of gaulic in old gildings by gaschromatography - mass spectrometry, Journal of chromatography, vol. 1107, 1-2, p. 226-232.

Castillejo, M. et al., 2002. Analytical study of the chemical and physical changes induced by $\mathrm{KrF}$ laser cleaning of tempera paints, Anal. Chem., 74 (18), p. 4662-4671.

Cennini, C. XIv siècle, (Déroche, C.), réédition 1991 - Le livre de l'art (Il libro dell'arte). Paris, Berger-Levrault, 408 p.

Chryssoulakis et Y., Chassery, J.-M., 1989. The application of physicochemical methods of analysis and image processing techniques to painted works of art, Athènes, University of Athens, $162 \mathrm{p}$.

Czerniak, V., Stouffs, J.-M., Tessariol, M. et Daniel, F., 2007. Le décor peint et la polychromie du portail occidental de la cathédrale Saint-Etienne de Cahors. Mémoire de la Société Archéologique du Midi de la France, Tome LXVII, Toulouse.

DE LA RIE, E. R., 1982. Fluorescence of paint and varnish layers (part I), Studies in Conservation, 27, p. 1-7.

DE LA RIE, E. R., 1982. Fluorescence of paint and varnish layers (part II), Studies in Conservation, 27, p. 65-69.

DE LA RIE, E. R., 1982. Fluorescence of paint and varnish layers (part III). Studies in Conservation, 27, p. 102-108.

Gaspard S., OujJa M., Moreno P., Méndez C., Garcia A., Domingo C., Castillejo M., 2008. Interaction of femtosecond laser pulses with tempera paints, Applied Surface Science, 255, p. 2675-2681.

KatsibIRI, O., 2004. Investigation of the gilding technique in two post-Byzantine wall paintings using micro-analytical techniques, Spectrochimica acta part B, 59, p. 1593-1599.

Larson, L., Shin, K. et ZinK, J., 1991. Photoluminescence Spectroscopy of Natural Resins and Organic Binding Media of Paintings, Journal of the American Institute for Conservation, 30 (1), p. 89-104.

Mallegol, J., Lemaire, J. et Gardette, J.-L., 2000. Long-term behaviour of oil-based varnishes and paints, Photo- and thermooxydation of cured linseed oil, Polymer degradation and Stability, 72, p. 191-197.

Mallegol, J., Lemaire, J. et Gardette, J.-L., 2001a. Yellowing of oil-based paints. Studies in conservation, 46 (2), p. 121-131.

MassChelein-KLeiner, L., 1992. Liants, vernis et adhésifs anciens, Bruxelles, Institut Royal du Patrimoine Artistique, $3^{\mathrm{e}}$ éd. 123 p.

Mounier, A., Daniel, F. et Bechtel, F., 2009a. Gilding techniques in mural paintings: three examples in the romanesque period in France. Proceedings of the $37^{\text {th }}$ International Symposium on Archaeometry, 12-16 Mai 2008, Sienne, Italie, sous presse.

Mounier, A, DANiel, F. et Bechtel, F., 2009b. L'illusion de l'or - Imitation de dorures dans les peintures murales médiévales. Communication orale, Colloque Aurum, Paris, C2RMF, 11-13 mai 2009.

Nevin, A. et Anglos, D., 2006. Assisted Interpretation of LaserInduced Fluorescence Spectra of Egg-Based Binding Media Using Total Emission Fluorescence Spectroscopy. Laser Chemistry, Volume 2006, ID 82823, 5 p.

Nevin, A., Cather, S., Anglos, D. et Fotakis, C., 2006. Analysis of protein-based binding media found in paintings using laser induced fluorescence spectroscopy. Analytica Chimica Acta, 573-574, p. 341-346.

Nevin, A., Comelli, D., Valentini, G., Anglos, D., Burnstock, A., Cather, S. et Cubeddu, R., 2007. Time-resolved fluorescence spectroscopy and imaging of proteinaceous binders used in paintings. Analytical and Bioanalytical Chemistry, 388, p. 1897-1905.

Nevin, A., Anglos, D., Cather, S. et Burnstock, A., 2008. The influence of visible light and inorganic pigments on fluorescence excitation emission spectra of egg-, casein- and collagenbased painting media, Applied Pysics A, 92, $8 \mathrm{p}$.

Nevin, A., Cather, S., Burnstock, A. et Anglos, D., 2008. Analysis of protein-based media commonly found in paintings using synchronous fluorescence spectroscopy combined with multivariate statistical analysis, Applied Spectroscopy, 62, 5, p. 481-489.

Perego, 2005. Dictionnaire des matériaux du peintre. Paris, Belin, $895 \mathrm{p}$.

Pline, $\mathrm{I}^{\mathrm{e}}$ siècle (réédition 1999, Zehnacker, H.). Histoire Naturelle, Chap. XXXIII, Nature des métaux, Paris, Les Belles Lettres, coll. "Classiques en poche".

Rabotyagova, O., Cebe, P. et Kaplan, D., 2008. Collagen structural hierarchy and susceptibility to degradation by ultraviolet radiation, Materials Science and Engineering C, 10p.

ArcheoSciences, revue d'archéométrie, 35, 2011, p. 19-28 
TheophILE (le moine), XII ${ }^{\mathrm{e}}$ siècle, (GUICHARD, J.-M., L'EsCALOPIER, C), réédition 1996. Essai sur divers arts, Nogent-Le-Roi, J. Laget, 314 p.

Thoury M., Elias, M., Frigerio, J.-M. et Barthou, C., 2007. Nondestructive Varnish Identification by Ultraviolet Fluorescence Spectroscopy, Applied Spectroscopy, 61, 12, p. $1275-1282$.

Toniolo, L., Colombo, C. Bruni, S. Fermo, P., Casoli, A., Palla, G. et Bianchi, C. L., 1998. Gilded Stuccoes of the Italian Baroque, Studies in Conservation, Vol. 43, 4, p. 201-208.
Watin, J.-F., 1773. L'art du peintre, doreur, vernisseur [etc.], Paris, Édition Grangé, $2^{\text {de }}$ édition, p. 141-171. [En ligne] site internet Gallica; [http://gallica2.bnf.fr/?lang=fr], consulté le 2 mai 2008.

Wisniewski, M., SionkowskaA, A., Kaczmarek, H., Lazare, S., Tokarev, V. et Belin, C., 2007. Spectroscopic study of a KrF excimer laser treated surface of the thin collagen films, Journal of Photochemistry and Photobiology A: Chemistry 188, p. 192-199. 